\title{
BIOBANKS. ASSOCIATED RISKS AND BENEFITS
}

Edmond Barcan ${ }^{1^{\#}}$, Stefan-Alexandru Artene ${ }^{1^{\#}}$, Anica Dricu ${ }^{1^{*}}$

\begin{abstract}
Biobanks are repositories of human organic material, that have an important role in the clinic. The World Health Organization (WHO) and the Global Health Ethics Unit have a great contribution considering the ideology of a well-functioning biobank. At the European Union, the functioning of biobanks is strictly regulated and research is being sustained by programs financed at a community level. The administration of biobanks brings both advantages and risks socially. To prevent these, international standards have been implemented for biobanks. In the research domain but also in the public health domain, an analysis in accordance with the legal framework must be considered in order to preserve the well-functioning of biobanks.
\end{abstract}

Keywords: Biobank, repository, guidelines, samples

\section{${ }^{1}$ University of Medicine and Pharmacy of Craiova, Faculty of Medicine, Romania "Corresponding author: Anica Dricu (anica.dricu@live.co.uk) \\ "Authors have contributed equally to this work Published online: 2 September 2020}

\section{Introduction}

Biobanks are mainly targeted at research organizations and teams, their function being as the base of organic material. The existence of these libraries also benefits the clinic, with the possibility for physicians to have a greater number of biological samples. These are useful in performing more complex analysis than those on samples already processed from patients. In addition to these benefits, the existence of biobanks also attracts ethical, moral and material obligations, established through contractual relations(1).

\section{Definitions. Types. Social impact}

Biobanks are constantly in the attention of the World Health Organization (WHO), especially because of their social importance. The Global Health Ethics Unit collaborates with various divisions at the WHO to develop mechanisms important in ethical surveillance, facilitating the collection, storage and dissemination of biological samples(2).

From the perspective of the Organisation for Economic Co-operation and Development (OECD), a biobank is defined as "a collection of biological material, along with the associated data and information"(3). Generally speaking, a 
biobank is set up to provide the necessary infrastructure for collecting, storing and administering biological material and related clinical data.

The International Society for Biological and Environmental Repositories (ISBER) defines biobanks as entities that acquire, store, process and/or disseminate biospecimens or samples when needed. The definition itself analytically includes geographic location functions of samples and specimens as well as activities associated with bank operations(4).

According to researcher AK Hawkins, the classification of biobanks is not simple and is based on different approaches. In the paper "Biobanks: Importance, Implications and Opportunities for Genetic Counselors", he claims that there are various types of biobanks, such as population-based, disease-oriented, hospital- or academic- based, networked or administered by government, non-profit or other legal entities(5).

In the paper "Biobank governance: trends and perspectives", researchers Gottweis and Zatloukal have described four types of research biobanks(6):

- Clinical/control based on biological specimen received from patients diagnosed with specific diseases and from non-diseased control;

- Population isolate biobanks that follow patients with similar genetics and that belong from analogous environments;
- Twin biobanks containing biological samples from monozygotic and dizygotic twins.

- Longitudinal population-based biobanks that track a portion of the population over an extended period of time;

A wider classification was provided by the team of researchers led by P. Rebulla in "Biobanking in the Year 2007"(7):

- Tissue collected during diagnostic procedure in clinic;

- Population biobanks;

- Organ biobanks;

- Disease biobanks that focus on a specific disease;

- Twin biobanks;

- Nonhuman biobanks.

Biobanks oriented around a specific disease are often hosted by a hospital with the purpose of collecting tumor and nontumor samples such as blood and other biological samples along with healthy tissue for comparison. By comparison, population biobanks are generally independent of hospitals, with samples being collected from apparently healthy individuals, not from patients. All types of biobanks are used to discover and study biomarkers, their type and relevance in fact dictating the nature of biobanks. Thus, disease susceptibility biomarkers may be detected in a population biobank, while exposure biomarkers or disease-specific biomarkers are easier to analyze from biobanks centered around a pathology(8). 
By their nature, the two types of biobanks are not mutually exclusive, because larger studies are not limited to just one type of information source. For example, if a study on pathophysiology and disease evolution reveals the existence of correlations of etiological nature in the phase when a disease does not manifest itself, researchers should resort to both types of biobanks(6). For this reason and others, it is necessary to develop structures that go beyond the classical barriers imposed by borders, geographic regions or ethnicity, giving birth to heterogeneous cyber networks such as the European initiative called the Biobanking and Biomolecular Resources Research Infrastructure (BBMRI).

At the EU level, the issue of biobanks has been scientifically addressed through the Council Decision of 19 December 2006 on the Specific Program "Cooperation" implementing the Seventh Framework Program of the European Community for Research, Technological Development and Demonstration Activities (2007-2013)(9). This activity aimed at developing and validating the tools and technologies needed to generate new knowledge, that was to be used into practical applications in the health and medicine field.

Through the research work, it has been pursued to catalyze the progress in the development of new research tools in modern biology, including in fundamental genomics, to significantly increase data generation and standardization improvement, acquisition and analysis of data and specimens through biobanks. Emphasis has been put on the development of new technologies needed at:

- Sequencing;

- Gene expression, genotyping and phenotyping;

- Structural and functional genomics, bioinformatics and systemic biology;

- Other technologies with the "omic" suffix.

For the 2014-2020 period, the European Commission has developed the Horizon 2020 Framework Program for Research and Innovation. The set of proposals for Horizon 2020, developed in full compliance with the Communication published by the Commission "A Budget for Europe 2020", fully supports the Europe 2020 Strategy. Since better understanding of illness and health is needed in people of all ages, it is necessary to develop new improved prevention, diagnosis and treatment measures(10).

Interdisciplinary, transferable pathophysiology research is essential to improve understanding of all aspects of disease processes, including a reclassification of normal variation and of disease based on molecular data, and also to validate and use research results in clinical applications. This requires close links between fundamental scientific research and clinical research and longterm cohort studies (and corresponding research fields). Closer links with research 
and medical infrastructures (databases, biobanks, etc.) are required for standardization, storage, sharing and access to data, which are all essential to maximize data use and to stimulate more innovative and more efficient ways of analyzing and combining data sets.

\section{Risks associated with biobanks}

At international level, the management and activity of biobanks is carried out in accordance with ISO 20387:2018(11).

Given that this standard has emerged for very little time, it is necessary that all institutions that administer biobanks comply with it, a process that will not be free of any problems or negative events.

ISO 20387:2018 specifies the general requirements that imply a biobank should have a competent, impartial and wellsustained operation, with emphasis put on the quality control requirements, which ensure that the biological material and data collections are made qualitatively. This standardization document is valid for all biobanking organizations, including biobanking for research and development of biological material from multicellular organisms (e.g. humans, animals, fungi and plants) and microorganisms.

Therefore, in case of lack of immediate and accurate compliance with ISO 20387:2018, there is a risk of direct threats to integrity or conformity of samples stored in biobanks. At the same time, the additional costs required to be committed to compliance will lead to costs not initially quantified, which complicates both the solvency of the legal entities involved and the relationship with patients or researchers. The fundamental elements that are affected by the occurrence of nonconformities in the functioning of biobanks are biosafety and biosecurity.

According to the $\mathrm{WHO}$, biosafety defines the set of principles, technologies and practices that are implemented to prevent unintended exposure to, or accidental release of pathogens and toxins. At the same time, biosecurity refers to institutional and personal security measures created to prevent the loss, theft, misuse, misappropriation or deliberate release of pathogens, genetically modified organisms, organisms that produce toxins or their parts, and of toxins that are held, or provided by the biobanks.

In this new context, the notion of biobank refers to the legal entity that provides biobanking services. These include both the activities of acquiring the ownership or custody of genetic materials or associated data as well as value-added services such as storage, together with activities related to the collection, preparation, preservation, testing, analysis and distribution of biological materials and related information. As such, the economic phenomenon associated with scientific research becomes dominant, with the risk of default becoming more and more likely.

It is also necessary to mention that until a sufficient number of evidences has been 
reached, a number of years may pass, a period in which we cannot even talk about financial sustainability. In this period and even after establishing a sufficiently large biorepository, financial support from an academic or medical institution is absolutely vital(8, 12, 13).

From the desire to achieve spectacular results in the long term, valuable resources are implemented in researching a biomarker that after years of ongoing research makes no contribution to the diagnosis or treatment of a disease. This leads to the accumulation of biological samples in the repository of limited or nonexistent value, representing an unjustified additional consumption of resources.

In addition to the risks associated with external factors, such as legal or regulatory changes, there are also risks directly associated with the internal factors of the organizations managing the banks. Such risks are both of human resources and functional nature, which include the organization's inadequacy in management changes or against the action of administrative or logistical threats.

An important gravity is held to the risks associated with human resources, such as the human error or the inadequacy of the equipment used. This can be mainly manifested during manipulation of biological samples or their subsequent data, with a negative impact on the consistency or integrity of stored samples or data. At the same time, errors in the
GDPR sphere can cause harm to people who have been guaranteed anonymity(14).

An important issue for those in charge of maintaining a biobanks and researchers using directly the evidence stored in the biobanks, and less for the clinicians and surgeons who handle them, is the quality of the biological material. A concrete example is that of the latency time between the time when the ischemia is installed in the tissue to be harvested and its proper sampling and storage $(15,16)$.

For those involved in researching and storing the sample, this time is crucial because of the sensitivity of certain proteins or metabolites to long-term exposure to hypoxia, but these aspects are minor details for a surgeon whose main concern is the patient's favorable progression. To avoid such situations, it is necessary to develop sampling and transport protocols in accordance with the sensitivity of each tissue type and good communication between all parties involved, from the sampler to those who deal with the indefinite storage of samples $(17,18)$.

A very important aspect of organizing a biobank is the protection offered both to donors and to those who manipulate or use the data in a biorepository. For patients, it is vital that the personal data they provide at the time of collection of the samples remains confidential. Otherwise, information may accidentally get into the hands of companies or people interested in making a profit or can be sold, with the 
privacy of patients being threatened. There have been several attempts to encrypt and protect data such that it is impossible to access personal data from anyone outside the biobank scheme. A nomenclature proposed by the European Medicines Authority has been adopted, bringing together the relevant authorities in Europe, the United States and Japan(19).

Sample analysis is governed by ISO 15189(20), the occurrence of noncompliance at the level of the biobank's management institution may lead to the emergence of bio-hazard phenomena. At the same time, the rules of protection are governed by ISO 15190(21), failure to comply with them leading to the risk of personal infection or the environment with noxious biological agents or the unauthorized dissemination of high-risk biohazard waste.

\section{Benefits brought by banks}

The emergence of the biobanks concept has led to the development of gene banks that incorporate multiple subtypes: seed bank, tissue bank, cryo-bank (sperm and egg bank). The preservation of biological samples is their main function, storing the genetic material of the samples for a long time, with utility in the restocking campaigns. In addition, biobanks have a very important role in the correction and maintenance of plant varieties and animal breeds.
The storage of biological samples and materials, as well as associated data in biobanks, provides researchers with the opportunity to take samples for their use in order to study the pathophysiology of certain diseases; obtaining treatments, markers of diagnosis and prognosis. This process, in itself, facilitates the acceleration of the understanding of the processes that characterize certain pathologies, the translational research having the biobanks as a substrate.

The fight against HIV/AIDS and against cancer is mainly supported by biobanks with human genetic material, which are the basis of support for the research institutes in the field. For example, the American Society of Clinical Oncology (ASCO) is based on the resources of thousands of biobanks, with the fight against cancer being limited by individual genetic characteristics, with no link between individuals and populations identified so far. Similarly, such activities are conducted by Cancer Research UK, but also individually.

The world population has recorded an exponential growth in recent decades, being projected at 9.6 billion people in 2050, compared with 7.6 billion today(22). A consequence of this upward trend due to several factors has been the increase in the number of cancer cases globally. Currently, cancer is ranked No. 2 worldwide among the causes of death, predicting that it will soon overcome cardiovascular disease and thus become 
the leading cause of death in the world(23, 24).

An important aspect of epidemiology in cancer is the disproportion between different ages, races, ethnic groups, sexes or risk groups and the incidence and mortality of this disease. However, with time, it has been observed that, despite epidemiological similarities, the evolution of cancer can be very different between two individuals sharing the same characteristics.

Moreover, the more in-depth study of individual genetic factors and the emergence of new branches such as metabolomics, proteomics, epigenomics have changed the vision of the way cancer develops, evolves and responds to each individual's treatment. Attention has moved to a molecular level where an impressive number of biomarkers can dramatically influence the course of the disease.

Thus, keeping the biological samples of the patients became crucial for the study and understanding of the complicated mechanisms behind the onset and progression of the disease, as well as for the formulation of a therapeutic decision especially according to the particularities of each patient. The retention of these bio samples has become a common practice, only in the United States their number being tripled over a decade, reaching about 600 million(25).

Biobanks therefore have the role of rescuing and preserving genetic resources, defending life. On this background, stem cell banks have grown and developed, with more and more people turning to the counter-offerings offered by them.

At the local level, the benefits of such institutions are reflected in the socioeconomic environment, through the creation of a labor force, and also by providing people with a chance to live, from the very beginning of extrauterine life. Taking stem cells at birth helps avoid the difficulty of finding a compatible donor in case of an incurable condition. Stem cells can be used by the child who is born, as well as by close family members (brother/sister, parents, compatible relative)(26).

\section{Conclusions}

Organizing and functioning of biobanks is done only by applying the legal and normative framework, since 2018 existing an international reference standard. The risk of depreciation of biological samples is directly proportional to the need for material and financial resources for the maintenance of the activity. For these reasons, a large number of biobanks closed down, unable to support themselves.

As a further development, a SWOT analysis on the operation and management of a local biobank will be taken into account. This study will seek to identify strategies or action plans that minimize the effects of identified risks and threats. 


\section{Funding}

This work was supported by Grant PN-II-

ID-PCE-2011-3-1041

\section{Conflict of interest}

The authors declare no conflict of interest.

\section{References}

1. Artene, S.-A., et al., Biobanking in a constantly developing medical world. The Scientific World Journal, 2013. 2013.

https://doi.org/10.1155/2013/343275

2. Organization, W.H., About ethics and health. 2018.

3. OECD, Creation and Governance of Human Genetic Research Databases. 2006.

4. 2012 best practices for repositories collection, storage, retrieval, and distribution of biological materials for research international society for biological and environmental repositories. Biopreserv Biobank, 2012. 10(2):p.79-161.

https://doi.org/10.1089/bio.2012.1022

5. Hawkins, A.K., Biobanks: importance, implications and opportunities for genetic counselors. J Genet Couns, 2010.19(5):p.423-9

https://doi.org/10.1007/s10897-010-9305-1

6. Gottweis, H. and K. Zatloukal, Biobank governance: trends and perspectives. Pathobiology, 2007. 74(4): $p$. 206-11.

https://doi.org/10.1159/000104446

7. Rebulla, P., et al., Biobanking in the year 2007. Transfusion Medicine and Hemotherapy, 2007. 34(4): p.286-292.

\section{https://doi.org/10.1159/000103922}

8. Riegman, P.H., et al., Biobanking for better healthcare. Molecular Oncology, 2008. 2(3): p. 213-222. https://doi.org/10.1016/j.molonc.2008.07.004

9. Beltran, P.J., et al., Ganitumab (AMG 479) inhibits IGF-II-dependent ovarian cancer growth and potentiates platinum-based chemotherapy. Clin Cancer Res, 2014. 20(11): p. 2947-58. https://doi.org/10.1158/1078-0432.CCR-13-3448

10. Orizont 2020. 2013, Comisia Europeană: Bruxelles.
11. Richardson, D.L., et al., Paclitaxel With and Without Pazopanib for Persistent or Recurrent Ovarian Cancer: A Randomized Clinical Trial. JAMA Oncol, 2018. 4(2): p. 196-202. https://doi.org/10.1001/jamaoncol.2017.4218

12. Bevilacqua, G., et al., The role of the pathologist in tissue banking: European Consensus Expert Group Report. 2010, Springer. https://doi.org/10.1007/s00428-010-0887-7

13. Hainaut, P., et al., Pathology as the cornerstone of human tissue banking: European consensus expert group report. Biopreservation and biobanking, 2009. 7(3): p. 157-160. https://doi.org/10.1089/bio.2010.7303

14. Regulation (EU) $2016 / 679$ of The European Parliament and of The Council of 27 April 2016 on the protection of natural persons with regard to the processing of personal data and on the free movement of such data, and repealing Directive 95/46/EC (General Data Protection Regulation). 2016, Official Journal of the European Union: Bruxelles.

15. Spruessel, A., et al., Tissue ischemia time affects gene and protein expression patterns within minutes following surgical tumor excision. Biotechniques, 2004. 36(6):p.1030-1037. https://doi.org/10.2144/04366RR04

16. Blackhall, F.H., et al., Stability and heterogeneity of expression profiles in lung cancer specimens harvested following surgical resection. Neoplasia, 2004.6(6):p.761-767.

https://doi.org/10.1593/neo.04301

17. Morente, M.M., et al., TuBaFrost 2: standardising tissue collection and quality control procedures for a European virtual frozen tissue bank network. European journal of cancer, 2006. 42(16): p. 26842691. https://doi.org/10.1016/j.ejca.2006.04.029

18. Biological, I.S.f. and E. Repositories, Collection, storage, retrieval and distribution of biological materials for research. Cell Preservation Technology, 2008.6(1):p.3-58. https://doi.org/10.1089/cpt.2008.9997

19. Guideline, I.H.T., Definitions for Genomic Biomarkers, Pharmacogenomics, Pharmacogenetics, Genomic Data and Sample Coding Categories E15. 2007.

20. Awasthi, N. and R.E. Schwarz, Profile of nintedanib in the treatment of solid tumors: the evidence to date. Onco Targets Ther, 2015. 8: p. 3691-701. https://doi.org/10.2147/OTT.S78805

21. Matei, D., et al., Activity of sorafenib in recurrent ovarian cancer and primary peritoneal carcinomatosis: 
a gynecologic oncology group trial. J Clin Oncol, 2011. 29(1):p.69-75.

https://doi.org/10.1200/JCO.2009.26.7856

22. DeSA, U., World population prospects: the 2012 revision. Population division of the department of economic and social affairs of the United Nations Secretariat, New York, 2013.

23. Weir, H.K., et al., Peer Reviewed: Heart Disease and Cancer Deaths-Trends and Projections in the United States, 1969-2020. Preventing chronic disease, 2016. 13.

https://doi.org/10.5888/pcd13.160211
24. Tofield, A., Cancer overtakes cardiovascular disease as the main cause of death in 12 European Union countries. 2016, Oxford Univ Press Great Clarendon St, Oxford Ox2 6dp, England.

25. Vaught, J., et al., Biobankonomics: developing a sustainable business model approach for the formation of a human tissue biobank. JNCl Monographs, 2011. 2011(42):p.24-31.

https://doi.org/10.1093/incimonographs/lgr009

26. Dricu, A., Recent challenges with stem cell banking. 2018, Taylor\&Francis.

https://doi.org/10.1080/14712598.2018.1445715 